CERN-TH/96-259

October 10, 1996

\title{
BARYON-TO-ENTROPY RATIO IN VERY HIGH ENERGY NUCLEAR COLLISIONS
}

\author{
K.J.Eskola ${ }^{\mathrm{a}, \mathrm{b}}$ and K. Kajantie $\mathrm{a}^{\mathrm{a}, \mathrm{b}} \mathrm{f}$ \\ a CERN/TH, CH-1211 Geneve 23, Switzerland \\ b Department of Physics, P.O.Box 9, 00014 University of Helsinki, Finland
}

\begin{abstract}
We compute as a function of rapidity $y$ the baryon number carried by quarks and antiquarks with $p_{\mathrm{T}}>p_{0} \approx 2 \mathrm{GeV}$ produced in $\mathrm{Pb}+\mathrm{Pb}$ collisions at $\mathrm{TeV}$ energies. The computation is carried out in lowest order QCD perturbation theory using structure functions compatible with HERA results. At $p_{0}=2 \mathrm{GeV}$ the initial gluon density is both transversally saturated and thermalised in the sense that the energy/gluon equals to that of an ideal gas with the same energy density. Even at these high energies the initial net baryon number density at $y=0$ at $\tau=0.1 \mathrm{fm}$ will be more than the normal nuclear matter density but the baryon-to-entropy ratio is only $(B-\bar{B}) / S \sim 1 / 5000$. Further evolution of the system is discussed and the final baryon-to-entropy ratio is estimated.
\end{abstract}

\footnotetext{
${ }^{1}$ kari.eskola@cern.ch

${ }^{2}$ keijo.kajantie@cern.ch
} 


\section{Introduction}

The formation of QCD plasma in central collisions of large nuclei in the $\mathrm{TeV}$ energy range can be described as the formation of a large number of partons (gluons and quarks) with transverse momentum $p_{\mathrm{T}}>p_{0}$, where $p_{0}$ parametrises the lower limit of the $p_{\mathrm{T}}$ of the partons included in the computation [1]. The number of these partons in an average collision can be estimated in QCD perturbation theory [2], [3]. It scales $\sim A^{4 / 3}$ and for $p_{0} \approx 2 \mathrm{GeV}$ its value is easily $\mathcal{O}(1000)$ per unit of rapidity in the central rapidity region. This process takes place in a time $1 / p_{0} \approx 0.1 \mathrm{fm} / \mathrm{c}$ and it is thus equal to the initial formation of QCD plasma. Making predictions for the final observables requires modelling the further evolution of the system either hydrodynamically [1, 5, 6] or in various kinetic models [7]- [13].

For a thermal system the computation of the overall multiplicity of the initial partons gives the entropy, associated with the temperature $T(t, \mathbf{x})$, and a computation of the quark and antiquark number gives the net quark number, associated with the chemical potential $\mu(t, \mathbf{x})$. The purpose of this article is to compute in more detail the initial amounts of gluons, quarks and antiquarks with $p_{\mathrm{T}}>p_{0}$ and from this to estimate the final baryon-to-entropy ratio.

At LHC energies, $\sqrt{s}=5500 \mathrm{GeV}$, the gluons are by far the dominant component of the initial state. In fact, for $p_{0}=2 \mathrm{GeV}$ we shall see that QCD perturbation theory implies that the gluonic subsystem has two remarkable properties: it is both thermalised, in the sense that its energy/gluon is the same as in a thermally equilibrated ideal gas with the corresponding energy density, and transversally saturated [14, 10, 9] in the sense that the total transverse area occupied by the gluons equals the total nuclear area. Thus, although $p_{0}$ in principle is a parameter constrained by soft physics [16, 17], its value $2 \mathrm{GeV}$ gets a well defined dynamical significance in heavy ion collisions at very high energies.

After the initial production the system goes through various expansion and phase transition stages. A natural first assumption is to assume that the expansion is adiabatic, so that both the total entropy $S$ and the net baryon number $B-\bar{B}$ in a unit of rapidity are constant. This, in particular, implies that $E_{T}$ in a unit of rapidity decreases rapidly, the work done against expansion transfers $E_{T}$ to larger rapidities from the central unit. We shall analyse this decrease, as well as possible increase of $S$ and $B-\bar{B}$ due to dissipation and non-perturbative source terms in some detail.

Concerning $B-\bar{B}$, the system produced in pp collisions is different from the one in $A A$ collisions. In pp the color correlations (strings) between individual quarks (antiquarks) produced in the central rapidity and the (di-)quarks left in the fragmentation regions are essential for baryon production [12, 13, 9, 10]. The system is dilute and essentially free streaming, and the (net) baryon number remains mostly in the fragmentation regions, while the mid-rapidity quark(antiquark) usually becomes an ingredient of a meson. Therefore, in pp collisions the initially produced $q-\bar{q}$ distribution in the 
mid-rapidity range does not directly reflect the final net baryon distribution. In heavy ion collisions, however, the situation should be different. At very high energies, the produced parton system in the central rapidity region is extremely dense, and its further evolution depends rather on the secondary collisions of partons (and strings as well) than on the direct color correlations with the fragmentation regions. This dynamics is built in hydrodynamical expansion equations, which imply in the adiabatic case that $B-\bar{B}$ in a unit of rapidity is constant.

At $\sqrt{s}=5500 \mathrm{GeV}$ the total number of quarks and antiquarks will be seen to be initially only $10 \%$ of that of gluons. The net quark number is much less but still calculable. In fact, we estimate that the initial $(B-\bar{B}) / S \approx 1 / 5000$ at $\tau=0.1 \mathrm{fm}$ which then may increase by up to a factor 4 , while in the $\sqrt{s}=20 \mathrm{GeV}$ range numbers of the order of $1 / 20 \ldots 1 / 50$ have been observed [18, 19]. Our result is in qualitative agreement with the expectation that at very high energies the baryon number of the initial nuclei goes to large forward and backward rapidities leaving the central region essentially depleted of net baryon number [20, 21]. Experimental data at AGS $(\sqrt{s}=5$ $\mathrm{GeV})$ and SPS $(\sqrt{s}=17 \mathrm{GeV})$ 22] have shown that nuclei are much more efficient in stopping baryons than pp collisions and it has been recently argued in a gluon field model of baryons [23] that this would persist to LHC energies $(\sqrt{s}$ in the TeV range). Also effects on the baryon-to-entropy ratio due to event-by-event fluctuations of the net baryon number have been discussed recently [24].

This approach of computing gluon and quark production stretches QCD perturbation to its limits and the sources of error are obvious: next-to-leading order (NLO) corrections with the present acceptance criteria have not been computed, there are uncertainties associated with small- $x$ gluon shadowing and with modeling nuclear effects for gluons in general, the quantitative results will somewhat depend on the parameter $p_{0}$ defining the lower limit of the $p_{\mathrm{T}}$ of quarks and gluons included. We offer the results for what they are worth; they will anyway set the stage for further refinements.

\section{The formulas}

The lowest order (LO) formula for inclusive production of partons in pp collisions can be found e.g. in Refs. 225]. Our main focus is on the production of semihard partons, defined by $p_{\mathrm{T}}>p_{0}$. The cross section for each flavour $f$ can be written as follows:

$$
\begin{array}{rl}
\frac{d \sigma^{f}}{d y}\left(\sqrt{s}, p_{0}\right)=\int d p_{\mathrm{T}}^{2} & d y_{2} \sum_{\substack{i j \\
\langle k l\rangle}} x_{1} f_{i / p}\left(x_{1}, Q^{2}\right) x_{2} f_{j / p}\left(x_{2}, Q^{2}\right) \times \\
\times\left[\delta_{f k} \frac{d \hat{\sigma}^{i j \rightarrow k l}}{d \hat{t}}(\hat{t}, \hat{u})+\delta_{f l} \frac{d \hat{\sigma}^{i j \rightarrow k l}}{d \hat{t}}(\hat{u}, \hat{t})\right] \frac{1}{1+\delta_{k l}},
\end{array}
$$


where the fractional momenta of incoming partons are

$$
x_{1}=\frac{p_{\mathrm{T}}}{\sqrt{s}}\left(\mathrm{e}^{y}+\mathrm{e}^{y_{2}}\right) \text { and } x_{2}=\frac{p_{\mathrm{T}}}{\sqrt{s}}\left(\mathrm{e}^{-y}+\mathrm{e}^{-y_{2}}\right)
$$

the region of integration is

$$
p_{0}^{2} \leq p_{\mathrm{T}}^{2} \leq \frac{s}{4 \cosh ^{2} y} \quad \text { and } \quad \ln \left(\frac{\sqrt{s}}{p_{\mathrm{T}}}-\mathrm{e}^{-y}\right) \leq y_{2} \leq \ln \left(\frac{\sqrt{s}}{p_{\mathrm{T}}}-\mathrm{e}^{y}\right),
$$

where $y$ is fixed so that

$$
|y| \leq \ln \left(\frac{\sqrt{s}}{2 p_{0}}+\sqrt{\frac{s}{4 p_{0}^{2}}-1}\right)
$$

and the invariants at the parton level are

$$
\hat{t}=-p_{\mathrm{T}}^{2}\left[1+\mathrm{e}^{-\left(y-y_{2}\right)}\right] \text { and } \hat{u}=-p_{\mathrm{T}}^{2}\left[1+\mathrm{e}^{\left(y-y_{2}\right)}\right] .
$$

The sum over initial states includes all combinations of two parton species:

$$
i j=g g, g q, q g, g \bar{q}, \bar{q} g, q q, q \bar{q}, \bar{q} q, \bar{q} \bar{q}
$$

while the final states consist of all pairs (without a mutual exchange):

$$
\langle k l\rangle=g g, g q, g \bar{q}, q q, q \bar{q}, \bar{q} \bar{q} .
$$

The factor $1 /\left(1+\delta_{k l}\right)$ is a statistical factor for identical particles in the final state. In the initial state, three (anti)quark flavours are taken into accout: $q=u, d, s$, and, in the final state four (massless) flavours: $q=u, d, s, c$. In the running $\alpha_{s}\left(p_{\mathrm{T}}\right)$ we, accordingly, use $N_{f}=4$. Since

$$
\frac{d \hat{\sigma}^{i j \rightarrow k l}}{d \hat{t}}(\hat{t}, \hat{u})=\frac{d \hat{\sigma}^{j i \rightarrow k l}}{d \hat{t}}(\hat{u}, \hat{t})
$$

there are only eight different types of subprocess cross sections:

$$
\begin{array}{rl}
g g \rightarrow g g & g q \rightarrow g q \\
g g \rightarrow q \bar{q} & q_{i} q_{j} \rightarrow q_{i} q_{j}, \quad i \neq j \\
q_{i} q_{i} \rightarrow q_{i} q_{i} & q_{i} \bar{q}_{i} \rightarrow q_{j} \bar{q}_{j}, \quad i \neq j \\
q_{i} \bar{q}_{i} \rightarrow q_{i} \bar{q}_{i} & q_{i} \bar{q}_{i} \rightarrow g g,
\end{array}
$$

which can be found, e.g., in refs. 25].

For the parton densities $f_{j / p}$, we use the set GRV 94 LO 26 with a scale choice $Q=p_{\mathrm{T}}$. With this set we can consistently extend our lowest order computation even below $p_{\mathrm{T}}=2 \mathrm{GeV}$. 
Summation over final state parton flavours $g, u, d, s, c$ now gives the production cross sections $d \sigma^{g} / d y, d \sigma^{q} / d y, d \sigma^{\bar{q}} / d y$ of gluons, quarks and antiquarks with $p_{\mathrm{T}}>p_{0}$ in a pp collision. These are normalised so that

$$
\int d y \frac{d \sigma^{f}}{d y} \equiv 2 \sigma_{\text {hard }}^{f}\left(\sqrt{s}, p_{0}\right)
$$

and

$$
\sigma_{\text {hard }}^{g}\left(\sqrt{s}, p_{0}\right)+\sigma_{\text {hard }}^{q}\left(\sqrt{s}, p_{0}\right)+\sigma_{\text {hard }}^{\bar{q}}\left(\sqrt{s}, p_{0}\right) \equiv \sigma_{\text {hard }}\left(\sqrt{s}, p_{0}\right),
$$

where $\sigma_{\text {hard }}\left(\sqrt{s}, p_{0}\right)$ is the cross section for one (semi)hard collision and the factor 2 comes from two partons in one (semi)hard event. Since we will be especially interested in the central rapidity region, $|y| \leq 0.5$, let us also define for later use

$$
\sigma_{\text {hard }}^{f}\left(\sqrt{s}, p_{0},|y| \leq 0.5\right) \equiv \frac{1}{2} \int_{-0.5}^{+0.5} d y \frac{d \sigma^{f}}{d y}
$$

The next-to-leading (NLO) order corrections to eq.(11) can be large and UA1 minijet data indicates [2] that a $\mathrm{K}$ factor of about 2.5 is needed at those energies. NLO jet analysis shows [27] that at higher energies the factor is somewhat less, $\mathrm{K} \approx 1.5$. To get a conservative estimate we shall use $\mathrm{K}=1$.

After the cross sections are computed on the pp level, they are transformed to the number $\bar{N}$ of events of the type described by $\sigma$ in an average central (zero impact parameter) $\mathrm{A}+\mathrm{A}$ collision by multiplying by the nuclear overlap function $T_{\mathrm{AA}}(\mathbf{b})$ at $\mathbf{b}=0: \bar{N}=\sigma T_{\mathrm{AA}}(\mathbf{b}=0)$. For example, the number distributions of produced flavour $f$ are obtained as

$$
\frac{d N^{f}}{d y}=T_{A A}(\mathbf{b}=0) \frac{d \sigma^{f}}{d y},
$$

and the total number of partons of flavor $f$ within $|y| \leq 0.5$ as

$$
\bar{N}_{A A}^{f}\left(\mathbf{b}=\mathbf{0}, \sqrt{s}, p_{0}\right)=2 T_{A A}(\mathbf{0}) \sigma_{\text {hard }}\left(\sqrt{s}, p_{0},|y| \leq 0.5\right)
$$

Numerically $T_{\mathrm{AA}}(0) \approx A^{2} / \pi R_{A}^{2}$ and we shall use the value

$$
T_{\mathrm{PbPb}}(0)=\frac{32}{\mathrm{mb}}
$$

This procedure is equivalent to neglecting nuclear shadowing, i.e. assuming that $f_{i / A}(x)=A f_{i / p}(x)$. When estimating various quantities we shall further use

$$
R_{\mathrm{Pb}}=6.54 \mathrm{fm}, \quad \pi R_{\mathrm{Pb}}^{2}=134 \mathrm{fm}^{2}, \quad V_{i}=\pi R_{\mathrm{Pb}}^{2} \Delta y / p_{0}=13.4 \mathrm{fm}^{3},
$$

where $V_{i}$ is the initial volume at the time $\tau_{i}=1 / p_{0}$ (with $p_{0}=2 \mathrm{GeV}$ ) when the quarks and gluons were formed. Note that for the longitudinal width for the volume near the 
central rapidity, we have approximated $\Delta z \approx \tau_{i} \Delta y$, i.e. we have neglected effects of the longitudinal spatial spread of the beam-partons.

A lower limit for the parameter $p_{0}$ is obtained from the transverse saturation criterion [1, 14, 9]. The partonic subsystem is saturated if the total transverse area occupied by the produced partons $=\bar{N}_{A A}(\mathbf{b}=0) \pi / p_{0}^{2}$ is larger than the total nuclear area $=\pi R_{A}^{2}$. For $\mathrm{Pb}+\mathrm{Pb}$ and $\bar{N}_{\mathrm{PbPb}}(\mathbf{b}=0)=2 \sigma_{\text {hard }}\left(\sqrt{s}, p_{0},|y| \leq 0.5\right) T_{\mathrm{PbPb}}(\mathbf{0})$ this converts to

$$
\frac{\sigma_{\text {hard }}\left(\sqrt{s}, p_{0},|y| \leq 0.5\right)}{\mathrm{mb}}>67\left(\frac{p_{0}}{2 \mathrm{GeV}}\right)^{2} .
$$

The formulas required for the computation of $E_{\mathrm{T}}$-distribution in a hard collision with an acceptance region can be found in [2]. We define our acceptance through a "measurement function" $\epsilon(y)$, which will in the following be chosen as a step function

$$
\epsilon(y)= \begin{cases}1, & \text { if }|y| \leq 0.5 \\ 0, & \text { otherwise }\end{cases}
$$

In $\mathrm{LO}$ the (mini)jets are produced back-to-back in $\mathbf{p}_{\mathrm{T}}$, and the $E_{\mathrm{T}}$-distribution in the chosen acceptance window can now be defined for each flavor $f$ as

$$
\begin{aligned}
\frac{d \sigma^{f}}{d E_{\mathrm{T}}}=\frac{1}{2} \int d p_{\mathrm{T}}^{2} d y_{1} d y_{2} \sum_{\substack{i j \\
\langle k l\rangle}} x_{1} f_{i / p}\left(x_{1},\right. & \left.Q^{2}\right) x_{2} f_{j / p}\left(x_{2}, Q^{2}\right) \frac{1}{1+\delta_{k l}} \times \\
\times & \left\{\frac{d \hat{\sigma}^{i j \rightarrow k l}}{d \hat{t}}(\hat{t}, \hat{u}) \delta\left(E_{\mathrm{T}}-\left[\delta_{f k} \epsilon\left(y_{1}\right)+\delta_{f l} \epsilon\left(y_{2}\right)\right] p_{\mathrm{T}}\right)+\right. \\
& \left.+\frac{d \hat{\sigma}^{i j \rightarrow k l}}{d \hat{t}}(\hat{u}, \hat{t}) \delta\left(E_{\mathrm{T}}-\left[\delta_{f l} \epsilon\left(y_{1}\right)+\delta_{f k} \epsilon\left(y_{2}\right)\right] p_{\mathrm{T}}\right)\right\}
\end{aligned}
$$

with the normalisation

$$
\sum_{f} \int d E_{\mathrm{T}} \frac{d \sigma^{f}}{d E_{\mathrm{T}}}=\sigma_{\text {hard }}\left(\sqrt{s}, p_{0}\right) .
$$

As explained in [2], this formulation takes into account that of the two minijets produced in the hard collision, there may be one or two or none (of flavor $f$ ) within the acceptance window. It is straightforward to obtain the first $E_{\mathrm{T}}$-moment for each flavor $f$ in the hard collision,

$$
\begin{aligned}
& \sigma_{\text {hard }}\left(\sqrt{s}, p_{0}\right)\left\langle E_{\mathrm{T}}^{f}\right\rangle=\int d E_{\mathrm{T}} \frac{d \sigma^{f}}{d E_{\mathrm{T}}} \\
&=\int d p_{\mathrm{T}}^{2} d y_{1} d y_{2} \sum_{\substack{i j \\
\langle k l\rangle}} x_{1} f_{i / p}\left(x_{1}, Q^{2}\right) x_{2} f_{j / p}\left(x_{2}, Q^{2}\right) \frac{1}{1+\delta_{k l}} \times \\
& \times\left[\delta_{f k} \frac{d \hat{\sigma}^{i j \rightarrow k l}}{d \hat{t}}(\hat{t}, \hat{u})+\delta_{f l} \frac{d \hat{\sigma}^{i j \rightarrow k l}}{d \hat{t}}(\hat{u}, \hat{t})\right] p_{\mathrm{T}} \epsilon\left(y_{1}\right)
\end{aligned}
$$


i.e. basically as the integral of Eq. (1) over $y$ in the accepted $y$-region, weighted by $p_{\mathrm{T}}$.

In an $A A$ collision with an impact parameter $\mathbf{b}$, the average transverse energy carried by semihard partons of flavor $f$ to the acceptance region becomes then

$$
\bar{E}_{\mathrm{T}}^{f}\left(\mathbf{b}, \sqrt{s}, p_{0}\right)=T_{A A}(\mathbf{b}) \sigma_{\mathrm{hard}}\left(\sqrt{s}, p_{0}\right)\left\langle E_{\mathrm{T}}^{f}\right\rangle,
$$

where $T_{A A}(\mathbf{b}) \sigma_{\text {hard }}\left(\sqrt{s}, p_{0}\right)$ is the average number of semihard collisions (all $y$ ) and $\left\langle E_{\mathrm{T}}^{f}\right\rangle$ is the average transverse energy of the flavor $f$ at $|y| \leq 0.5$ in each semihard collision.

\section{The results}

The following results are based on a numerical evaluation of Eqs. (1) and (20). For definiteness, we give the results for all $y$, in spite of the fact that very large $|y|$ phenomena cannot be experimentally observed in the collider mode and in spite of the fact that they are theoretically less well founded than the results near $y=0$ - they involve structure functions at extremely small $x \approx p_{0}^{2} / s$, where they have not yet been measured, for nuclei, in particular.

Firstly, to give an idea of the subprocesses contributing to $g, q$ and $\bar{q}$ production, Fig. 1 gives the $y$ distributions from the various subprocesses at $\sqrt{s}=5500 \mathrm{GeV}$. The scale at left refers to pp collisions, that on the right, obtained by using Eqs.(12)-(14), gives the corresponding number of partons produced in an average central $\mathrm{Pb}+\mathrm{Pb}$ collision. This scaling from $\mathrm{pp}$ to $\mathrm{Pb}+\mathrm{Pb}$ is not valid for large $|y|$, where it may lead to a violation of energy or baryon number conservation. For kinematical reasons very small values of $x$ are involved (see Fig. 4 below) and shadowing becomes important. In Fig. 2 for $\sqrt{s}=200 \mathrm{GeV}$ one is far from violating energy or baryon number conservation in $\mathrm{Pb}+\mathrm{Pb}$ for $|y| \lesssim 4$ and one can thus apply the right hand scale at least up to this $y$; for pp there is no problem at any $y$.

One observes that for gluons the process $g g \rightarrow g g$ is dominant and increasingly so with increasing $\sqrt{s}$. For quarks the dominant process is $g q \rightarrow g q$ and the forwardbackward peaking is due to valence quarks in this subprocess. For antiquarks the dominant process is $g \bar{q} \rightarrow g \bar{q}$, without any valence quark contribution.

The $y$ distributions are summarised in Fig. 3, which also shows the important net quark number distribution. Some numerical values are given in Tables 1 and 2. 

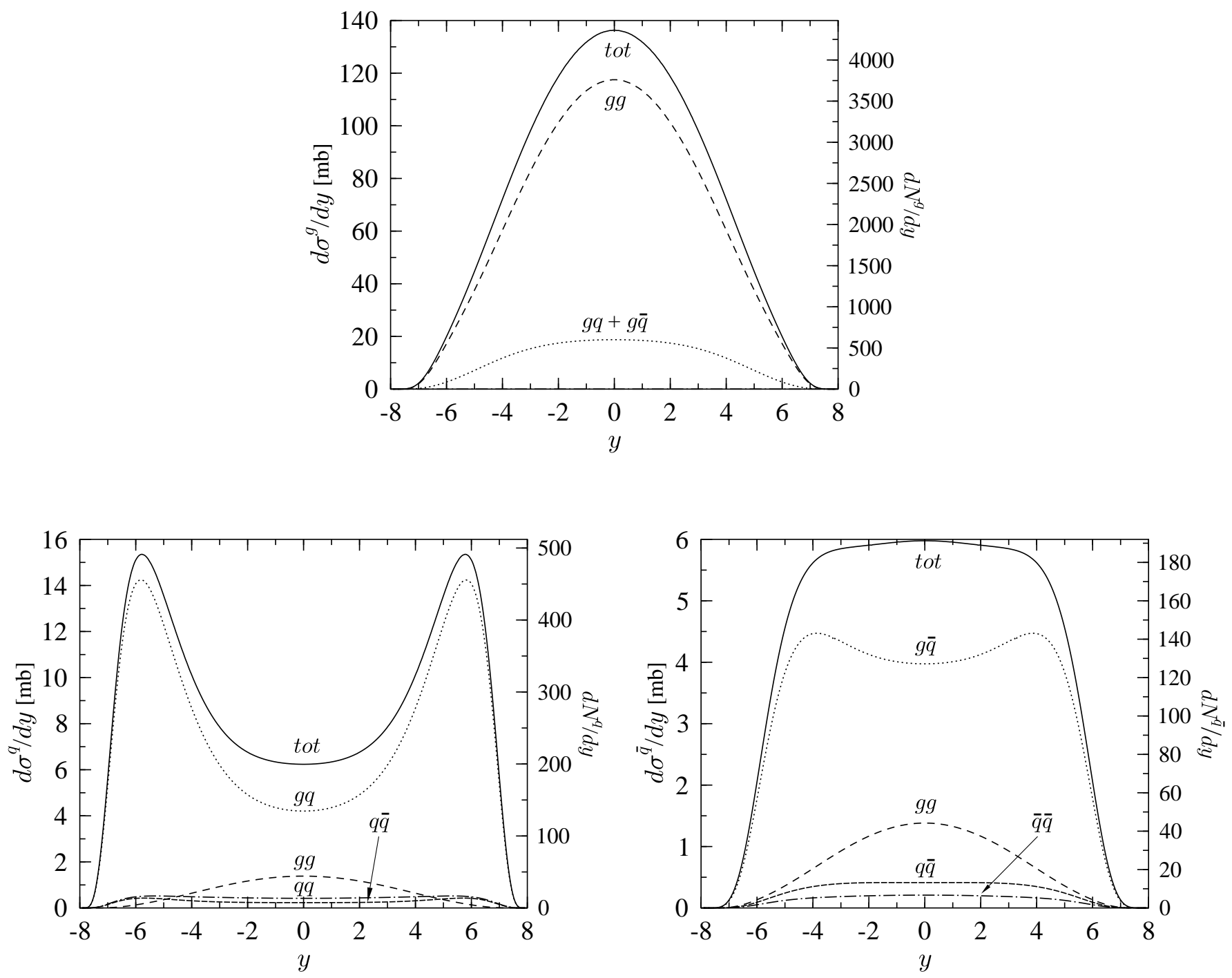

Figure 1: The rapidity distributions of gluons, quarks and antiquarks $\left(p_{\mathrm{T}}>p_{0}=2\right.$ $\mathrm{GeV}$ ) produced in pp collisions at $\sqrt{s}=5500 \mathrm{GeV}$ together with the subprocess contributions. The axis to the right (to be applied only for $|y| \lesssim 4$ ) gives the corresponding number of partons produced in an average central $\mathrm{Pb}+\mathrm{Pb}$ collision.

\subsection{Initial state}

On the basis of the previous results one can immediately discuss the appearance of the system at the initial time $\tau_{i}=0.1 \mathrm{fm}$. For concreteness we give fixed numbers neglecting possible errors - and keep even too many decimals. Due to the expected dominance of semihard processes in the TeV energy range, let us mainly focus on the 

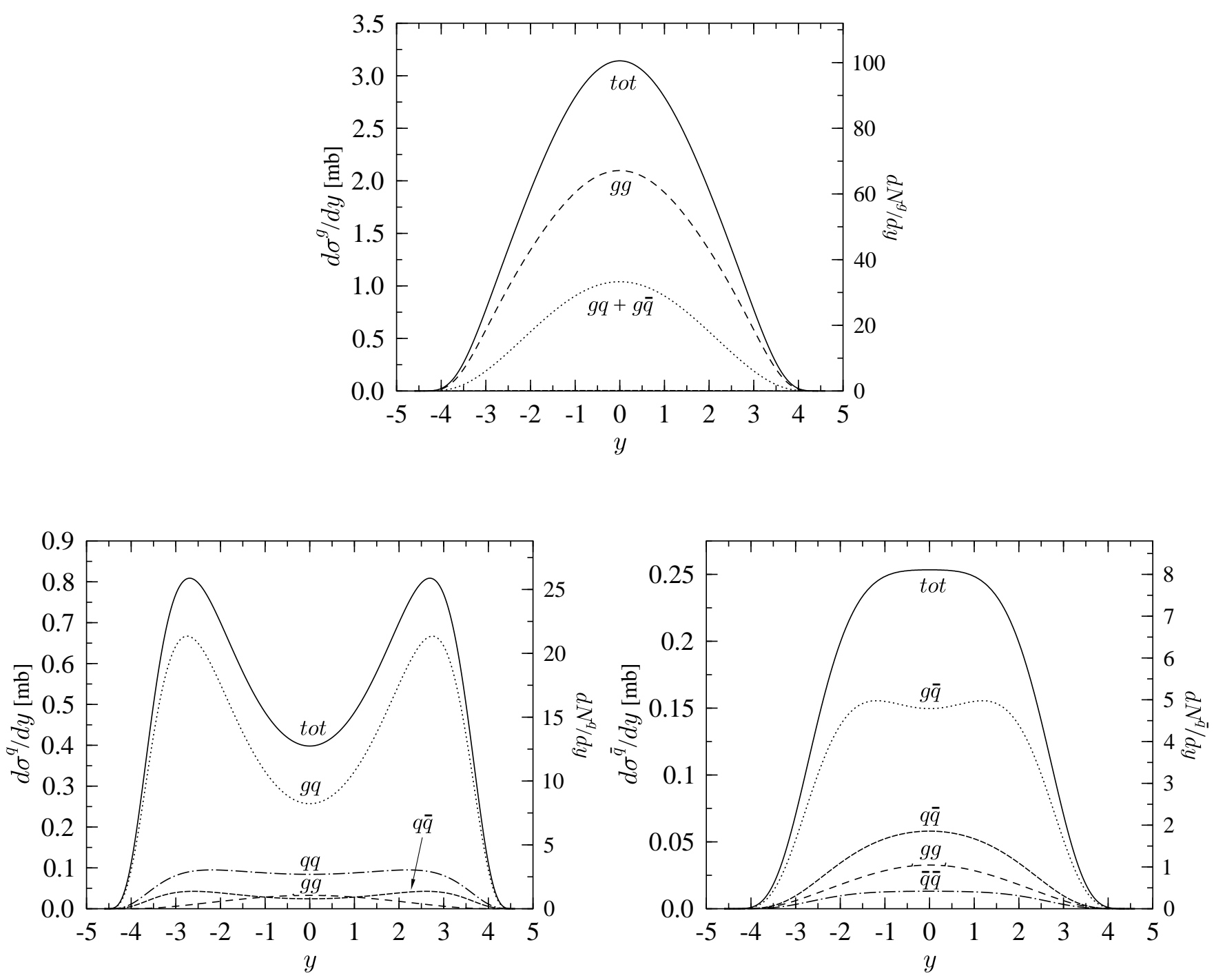

Figure 2: As Fig. 1 but for $\sqrt{s}=200 \mathrm{GeV}$

results for LHC. In the figures and in the tables, we give the numbers for $\sqrt{s}=200$ $\mathrm{GeV}$ for comparison, so that our discussion may be reproduced for RHIC as well. Thus, unless otherwise stated, the numbers apply to an average central $\mathrm{Pb}+\mathrm{Pb}$ collision at $\sqrt{s}=5500 \mathrm{GeV}, p_{0}=2 \mathrm{GeV}$ and the rapidity range $-0.5<y<0.5$. The results in Fig. 1 and Tables 1 and 2 firstly imply that there are

$$
4350 \text { gluons }+200 \text { quarks }+190 \text { antiquarks, }
$$

which carry a transverse energy of

$12960 \mathrm{GeV}$ for gluons $+620 \mathrm{GeV}$ for quarks $+590 \mathrm{GeV}$ for antiquarks. (23) 

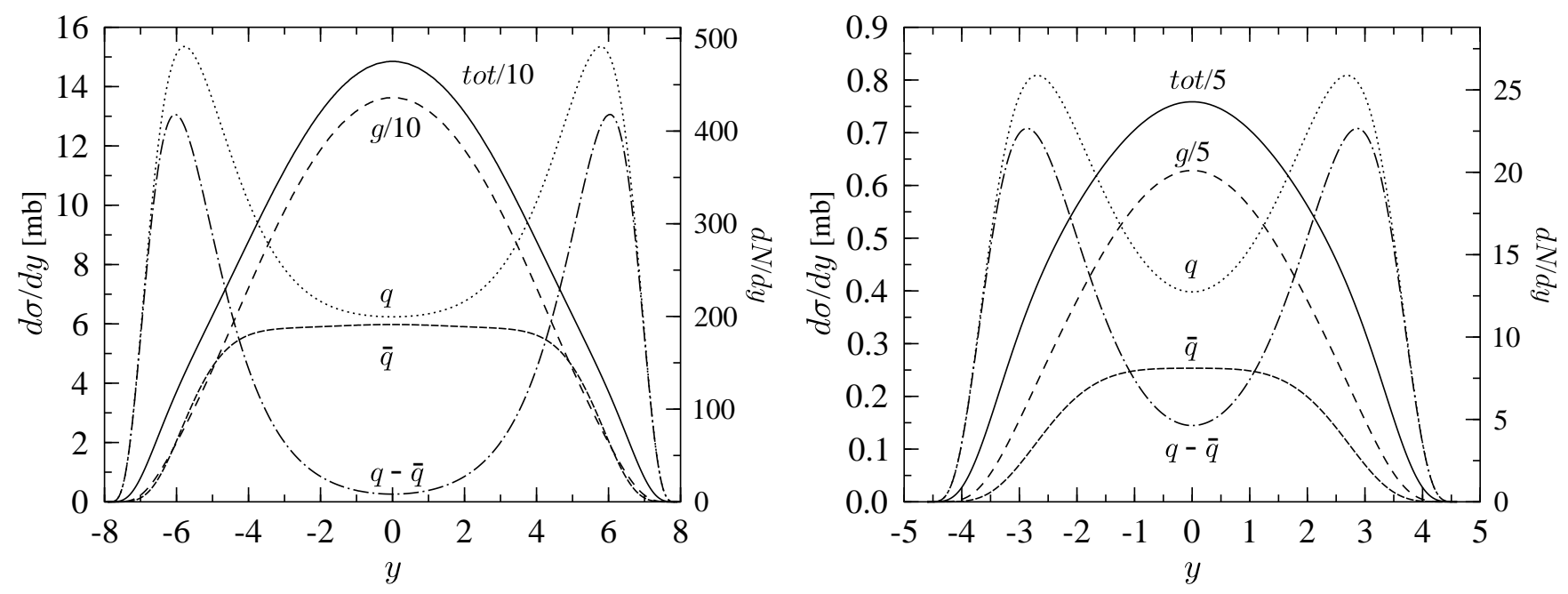

Figure 3: Distributions of various quanta for $\sqrt{s}=5500,200 \mathrm{GeV}$. Note that the total hard and gluon cross sections have been scaled down by a factor 10 and 5 for $\sqrt{s}=5500$ $\mathrm{GeV}$ and $200 \mathrm{GeV}$, respectively.

\begin{tabular}{|c|c|c|c|c|c|}
\hline$\sqrt{s} / A$ & range of $y$ & total & $g$ & $q$ & $\bar{q}$ \\
\hline 5500 & $|y|<0.5$ & 74.06 & 67.95 & 3.123 & 2.988 \\
& all $y$ & 657.5 & 555.8 & 68.34 & 33.06 \\
\hline \hline 200 & $|y|<0.5$ & 1.885 & 1.556 & 0.2025 & 0.1266 \\
& all $y$ & 10.00 & 7.077 & 2.278 & 0.6454 \\
\hline
\end{tabular}

Table 1: Values in mb of the perturbative parton cross sections $\sigma_{\text {hard }}^{f}\left(\sqrt{s}, p_{0}\right)$ and $\sigma_{\text {hard }}^{f}\left(\sqrt{s}, p_{0},|y| \leq 0.5\right)$ in pp collisions at $\sqrt{s}=5500 \mathrm{GeV}, p_{0}=2 \mathrm{GeV}$. The number of partons of type $f=g, q, \bar{q}$ in an average $\mathrm{Pb}+\mathrm{Pb}$ collision is $\bar{N}_{\mathrm{PbPb}}^{f}=2 \sigma_{\text {hard }}^{f} T_{\mathrm{PbPb}}(\mathbf{0}) \approx$ $64 \sigma_{\text {hard }}^{f}$.

In a space-time picture these are formed at a time $1 / p_{0}=0.1 \mathrm{fm}$ after the collision and thus, using eqs.(15), the corresponding number and energy densities are

$$
\begin{gathered}
n_{g}=\frac{325}{\mathrm{fm}^{3}}, \quad n_{q}=\frac{14.9}{\mathrm{fm}^{3}}, \quad n_{\bar{q}}=\frac{14.2}{\mathrm{fm}^{3}} \\
\epsilon_{g}=967 \frac{\mathrm{GeV}}{\mathrm{fm}^{3}}, \quad \epsilon_{q}=46.3 \frac{\mathrm{GeV}}{\mathrm{fm}^{3}}, \quad \epsilon_{\bar{q}}=43.9 \frac{\mathrm{GeV}}{\mathrm{fm}^{3}} .
\end{gathered}
$$

Note that here we consider an idealized (simplified) picture of a very high energy heavy ion collision, where the transit time of the colliding nuclei is reduced to zero. For the TeV energy range, this is a good approximation, since then $2 R_{\mathrm{Pb}} / \gamma \ll 1 / p_{0}$. However, semihard and softer gluons extend longitudinally much further than the strongly 


\begin{tabular}{|c|c|c|c|c|c|}
\hline$\sqrt{s} / A$ & range of $y$ & total & $g$ & $q$ & $\bar{q}$ \\
\hline 5500 & $|y|<0.5$ & 443 & 405 & 19.4 & 18.4 \\
& all $y$ & 3830 & 3227 & 407 & 196 \\
\hline \hline 200 & $|y|<0.5$ & 10.02 & 8.211 & 1.127 & 0.6834 \\
& all $y$ & 51.99 & 36.53 & 12.09 & 3.370 \\
\hline
\end{tabular}

Table 2: As Table 1 but for values of the first $E_{\mathrm{T}}$-moments $\sigma_{\text {hard }}\left\langle E_{\mathrm{T}}^{f}\right\rangle$ in mbGeV of the parton distribution. The transverse energy of partons of type $f$ in an average central $\mathrm{Pb}+\mathrm{Pb}$ collision is $\bar{E}_{\mathrm{T}}^{f}=\sigma_{\text {hard }}\left\langle E_{\mathrm{T}}^{f}\right\rangle T_{\mathrm{PbPb}}(\mathbf{0}) \approx 32 \sigma_{\text {hard }}\left\langle E_{\mathrm{T}}^{f}\right\rangle$.

contracted valence components. For the semihard partons, the typical uncertainty in the longitudinal direction is $\Delta z \sim 2 /\left(x_{1(2)} \sqrt{s}\right) \sim 1 / p_{0}$. This width in the production mechanism [28] is also neglected in the idealized picture. Then, in the central rapidity unit, one may estimate $\langle E\rangle \approx 2\left\langle E_{\mathrm{T}}\right\rangle \sinh 0.5=1.04\left\langle E_{\mathrm{T}}\right\rangle$ for the initial parton production, so that converting the transverse energy directly into energy density is a good approximation.

Let us briefly recapitulate the discussion of the gluonic subsystem [3], which has two notable properties. Firstly, the $E_{\mathrm{T}}$ per gluon is

$$
\frac{\bar{E}_{\mathrm{T}}^{g}}{\bar{N}_{\mathrm{PbPb}}^{g}}=\frac{\epsilon_{g}^{\mathrm{pQCD}}}{n_{g}^{\mathrm{pQCD}}}=2.98 \mathrm{GeV},
$$

which naturally is somewhat larger than $p_{0}$. For an ideal gas of massless gluons in a complete thermal equilibrium, the temperature can be determined from

$$
\epsilon_{g}^{\text {ideal }}=3 \pi^{2} / 90 \cdot 16 T_{\mathrm{eq}}^{4}
$$

For an ideal gas with $\epsilon_{g}^{\text {ideal }}=\epsilon_{g}^{\mathrm{pQCD}}$ we find $T_{\text {eq }}=1.10 \mathrm{GeV}$. It is interesting, and surprising, to see that

$$
\frac{\epsilon_{g}^{\mathrm{pQCD}}}{n_{g}^{\mathrm{pQCD}}} \approx \frac{\epsilon_{g}^{\text {ideal }}}{n_{g}^{\text {ideal }}}=2.7 T_{\text {eq }} .
$$

As far as energy per particle goes, the initial gluon subsystem thus is "thermalised" from the beginning. Note especially that we have not considered isotropisation of the system at all. In our idealized picture, $\left\langle p_{z}^{2}\right\rangle \ll\left\langle p_{\mathrm{T}}^{2}\right\rangle / 2$ initially in the central rapidity unit and collisions are needed to make the system isotropic. What our conclusion does indicate, however, is that since the appropriate energy/gluon is already there, no additional particle production is necessary, increasing thus possibilities for a rapid thermalisation. At this point it is perhaps worth mentioning that both the time of isotropisation and the conclusion of thermalisation depends on modelling of the initial collisions, especially on how the longitudinal width of initial parton production is accounted for. How rapidly 
isotropisation then proceeds depends on the expansion of the system and on the rate of secondary collisions. Note that if there exists a finite width in the longitudinal direction, one may estimate the isotropisation time (and the initial conditions) also in a free streaming picture [8, 28].

Secondly, with the present $p_{0}=2 \mathrm{GeV}$ the gluonic subsystem satisfies precisely the transverse saturation criterion (16) for $\sqrt{s}=5500 \mathrm{GeV}$. In general, for pp collisions, $p_{0}$ is a phenomenological parameter separating the computable perturbative physics from the more model dependent soft physics. The value of $p_{0}$ depends on what is assumed for the soft physics, and it is restricted by the measured total and inelastic cross sections [16] and transverse momentum spectra of charged particles in $\mathrm{p} \overline{\mathrm{p}}$ and pp collisions [17]. As we have shown above, sufficiently many semihard partons are produced in a heavy ion collision at very high energy, so that $p_{0}$ gets a better defined dynamical significance, indicating that the soft component becomes less relevant. From Table 1 we can see that there is no transverse saturation at $p_{0}=2 \mathrm{GeV}$ for $\sqrt{s}=200$ $\mathrm{GeV}$ but the saturation occurs for smaller $p_{0}$, involving $p_{\mathrm{T}}$ 's in the non-perturbative regime. In fact, saturation is reached at $p_{0}=1.6 \mathrm{GeV}$ for $\sqrt{s}=1.8 \mathrm{TeV}$ and at $p_{0}=1.0$ $\mathrm{GeV}$ for $\sqrt{s}=200 \mathrm{GeV}$. Therefore, at the RHIC energies, one should not neglect the soft component. A phenomenological way to obtain the saturation (dynamical screening) in a self-consistent manner in the initial parton production was suggested in Ref. [29].

Consider then the (anti)quarks, the density of which is below the thermal density by a factor of about 30. From Eq. 24) one sees that the initial net baryon number density at the time $1 / p_{0}=0.1 \mathrm{fm}$ is

$$
n_{B-\bar{B}} \equiv \frac{1}{3}\left(n_{q}-n_{\bar{q}}\right)=\frac{0.21}{\mathrm{fm}^{3}}
$$

Thus even at these ultrarelativistic energies the initial net baryon number density is more than the usual nuclear matter density, even if the initially baryon-rich fragmentation regions are already far apart in the forward and backward directions. Similarly, one may also compute the initial asymmetry:

$$
\begin{array}{rlrl}
\frac{B-\bar{B}}{B+\bar{B}} & =18 \% \ldots 29 \%, & & \sqrt{s}=200 \mathrm{GeV} \\
& =4.7 \% \ldots 6.3 \%, & \sqrt{s}=1800 \mathrm{GeV} \\
& =2.1 \% \ldots 2.6 \%, & \sqrt{s}=5500 \mathrm{GeV}
\end{array}
$$

where the range of values shown corresponds to varying $p_{0}$ from 1 to $3 \mathrm{GeV}$ and where the result also is given for lower energies for comparison. Note that the initial asymmetry depends only mildly on $p_{0}$. The difference between $\sqrt{s}=5500 \mathrm{GeV}$ and $200 \mathrm{GeV}$ is clearly due to the increase in the quark and antiquark rates with increasing energy, the difference $B-\bar{B}$ varying less rapidly. Although the mechanism considered here is perturbative, the initial asymmetry for the LHC energies is of the same order 
of magnitude as what is obtained in Ref. [23] in a purely non-perturbative gluon field model.

Finally, since for a thermal boson gas $s=3.60 n$, the total initial entropy (using the number of effectively thermal gluons) is $S=15900$ and, since $B-\bar{B}=2.9$ initially, the initial net baryon-to-entropy ratio at $\tau_{i}=0.1 \mathrm{fm}$ is

$$
\frac{B-\bar{B}}{S} \approx 1.8 \cdot 10^{-4} \sim \frac{1}{5000}
$$

It is also interesting to compare this number with the one for the early Universe, where the inverse of the specific entropy is $\sim 10^{-9}$. Thus we are still relatively far from the extreme conditions in the early Universe.

\subsection{Further evolution}

Further evolution of the system will go through possible thermalisation (quarks also may be thermalised), plasma expansion, phase transition, hadron gas expansion and decoupling stages. It is clear that any conclusions will at present be model dependent.

The simplest assumption is that of instant thermalisation and further essentially adiabatic expansion during all the stages, in analogy with the early universe. If the entropy $(S \approx 15900)$, net baryon number $(B-\bar{B} \approx 3)$ and the net baryon number-toentropy ratio $((B-\bar{B}) / S=1 / 5000)$ are all conserved, the initial values are also the final ones. For a hadron gas $S / N \gtrsim 4$ depends somewhat on the decoupling temperature. For a pion gas $S \approx 4 N$ and a multiplicity of about 4000 is predicted.

The assumption of instant thermalisation for quarks is, of course, unrealistic. Instead, one may assume that they thermalise adiabatically at some later time $\tau_{q}$. From the constancy of entropy it then follows that $g_{g} T^{3}=\left(g_{g}+g_{q}\right) T_{\text {new }}^{3}$, i.e.,

$$
T_{\text {new }}=T\left(1+g_{q} / g_{g}\right)^{-1 / 3}
$$

the increase $g_{g} \rightarrow g_{g}+g_{q}$ in the number of thermalised degrees of freedom is compensated for by a decrease in $T$. The total entropy and the multiplicity prediction is unchanged, but the energy density is decreased by the same factor as $T$. Note that the adiabatic scenario of quark thermalisation leads to a lower limit of the final pion multiplicity. Non-equilibrium models, where entropy, and therefore also the pion multiplicity, increase due to the chemical equilibration of quarks have also been presented 8.

Assuming that each produced particle carries on the average a transverse energy of $m_{T} \approx 0.5 \mathrm{GeV}$, the final transverse energy in the thermal scenario is about $2000 \mathrm{GeV}$. What happened to the large initial energy of $13000 \mathrm{GeV}$ in Eq.(25) in this scenario? It has been transmitted to larger rapidities by the work done against longitudinal expansion. In other words, the total entropy and number of particles in a unit of 
rapidity remain constant, but since the energy/particle $\sim T \sim 1 / \tau^{1 / 3}$ decreases, the total energy in a unit of rapidity decreases. The total reduction factor is the ratio between initial and final values of energy $/$ particle $=2.7 T_{i} / m_{T} \approx 6$.

We thus come to a series of scenarios starting from fully adiabatic expansion proceeding via increased amount of dissipation to a free-streaming expansion. The predictions of the final multiplicity and $E_{\mathrm{T}}$ starting from the same initial values in eqs. (24,25) thus can vary from $N_{\text {fin }}=4000, E_{\text {Tfin }}=2000 \mathrm{GeV}$ to values larger than these by a factor of up to six. To narrow this range, reliable estimates for mean free paths would be needed.

It may be of some interest to show how increasing dissipation can be described by the introduction of a shear viscosity [30, 田. If one writes $\eta=\eta_{0} T^{3}$ and uses $\epsilon=3 a T^{4}$, the solution of the usual longitudinal hydrodynamical equations for $T$ is

$$
\begin{aligned}
T & =T_{i}\left(\frac{\tau_{i}}{\tau}\right)^{1 / 3}+\frac{\eta_{0}}{3 a} \frac{1}{2 \tau_{i}}\left[\left(\frac{\tau_{i}}{\tau}\right)^{1 / 3}-\frac{\tau_{i}}{\tau}\right] \\
& \rightarrow\left[T_{i}+\frac{\eta_{0}}{6 a \tau_{i}}\right]\left(\frac{\tau_{i}}{\tau}\right)^{1 / 3} .
\end{aligned}
$$

Dissipation thus increases the temperature one reaches at a certain proper time and thus it also increases the lifetime and the multiplicity. All of this is parametrised by $\eta_{0}$. By definition, the effect must be small, $\eta_{0} \ll 6 a T_{i} \tau_{i}$. In the present context, $\eta_{0}$ could, in principle, be measured by observing deviations from the adiabatic multiplicity of about $N_{\text {fin }}=4000$. In practice, however, this leading estimate is not reliable enough to permit measurements of corrections to it.

\section{Soft component?}

One of the motivations for the choice of taking $p_{0}=2 \mathrm{GeV}$ was that at this value the produced gluons are so numerous that they are transversally saturated (Eq.(16)) so that [1] this semihard component gives a quantitatively correct estimate of an average event at LHC energies. At lower energies the saturation is reached for still smaller values of $p_{0}$ (at $p_{0}=1.6 \mathrm{GeV}$ for $\sqrt{s}=1.8 \mathrm{TeV}$, at $p_{0}=1.0 \mathrm{GeV}$ for $\sqrt{s}=200 \mathrm{GeV}$ ), for which next-to-leading order corrections are expected to be larger. At SPS energies,

$\sqrt{s}=20 \mathrm{GeV}$, the whole approach becomes useless. At these energies the bulk of the events is described by a nonperturbative soft component based on string or colour field formation and decay.

A way to assess the importance of the corrections is to assume that the soft component, dominant at $\sqrt{s}=20 \mathrm{GeV}$, is constant [16. The SPS data then imply 31 that this soft component gives an average contribution of $\bar{E}_{\mathrm{T}}^{\text {soft }}(\mathbf{0}) \approx 420 \mathrm{GeV}$ in the central rapidity unit of central $\mathrm{Pb}-\mathrm{Pb}$ collisions. The corresponding perturbative number $\bar{E}_{\mathrm{T}}^{\text {hard }}(\mathbf{0})$ is $14200 \mathrm{GeV}$ at $\sqrt{s}=5500 \mathrm{GeV}\left(p_{0}=2.0 \mathrm{GeV}\right.$, Table 2$)$. With these 
numbers, (assuming that for the SPS the hydrodynamical stage of the system is not very long) a simple order-of-magnitude estimate for the final baryon-to-entropy ratio can be made. Let us decompose the ratio into soft and hard contributions:

$$
\frac{B-\bar{B}}{S}=p_{\text {hard }}\left(\frac{B-\bar{B}}{S}\right)_{\text {hard }}+p_{\text {soft }}\left(\frac{B-\bar{B}}{S}\right)_{\text {soft }},
$$

where $p_{\text {hard }}=S_{\text {hard }} / S$ and $p_{\text {soft }}=S_{\text {soft }} / S$. An estimate for these is obtained from the transverse energies above,

$$
p_{\text {hard }} \approx \frac{\bar{E}_{\mathrm{T}}^{\text {hard }}}{\bar{E}_{\mathrm{T}}^{\text {hard }}+\bar{E}_{\mathrm{T}}^{\text {soft }}}, \quad p_{\text {soft }} \approx \frac{\bar{E}_{\mathrm{T}}^{\text {soft }}}{\bar{E}_{\mathrm{T}}^{\text {hard }}+\bar{E}_{\mathrm{T}}^{\text {soft }}} .
$$

Using then the baryon-to-entropy ratio as in Eq. (31) for the hard component, and 1/50 from Ref. [18] for the soft component, we obtain the final baryon-to-entropy ratio

$$
\frac{B-\bar{B}}{S} \sim 8 \cdot 10^{-4}
$$

for nuclear collisions at LHC.

For RHIC, the corresponding estimate is more unreliable due to the following reasons: Firstly, as discussed above, at $\sqrt{s}=200 \mathrm{GeV}$, the saturation of minijet production occurs at $p_{\mathrm{T}} \sim 1.0 \mathrm{GeV}$ but as this may already be in the region where the minijet cross sections become unreliable, let us consider a range from $p_{0}=1.5 \mathrm{GeV}$ to 2.0 $\mathrm{GeV}$. This gives $\bar{E}_{\mathrm{T}}^{\text {hard }}(\mathbf{0})=708 \mathrm{GeV}$ to $326 \mathrm{GeV}$. Secondly, although the gluons do dominate the early parton system also at RHIC, the energy/gluon is not thermal, so the assumption of instant thermalisation of glue at $\tau \sim 0.1 \mathrm{fm}$ is probably not as good as for the LHC. Following, nevertheless, the same line of reasoning as for the LHC gives $(B-\bar{B}) / S \sim 2 \cdot 10^{-3}$ initially at $\tau \sim 0.1 \mathrm{fm}$, and $(B-\bar{B}) / S \sim 9 \cdot 10^{-3}$ for the final baryon-to-entropy ratio for nuclear collisions at RHIC.

We see that the nonperturbative sources may increase the baryon-to-entropy ratio considerably, by a factor of about 4, from the initial values both at LHC and RHIC. At the LHC, the increase is dominantly due to the increase in the net baryon number and not in the total entropy, while at the RHIC both the net baryon number and the total entropy are increased by the soft source contributions.

\section{$5 \quad$ Very forward and backward partons}

The previous discussion has been confined to the $y=0$ region, although the curves in Figs. 1-3 are computed over the entire rapidity range. They are reliable on the pp level, but the simple transformation in Eqs. (12,14) used to transform them to the $\mathrm{A}+\mathrm{A}$ level assumes independent subcollisions and thus does not take into account energy and baryon number conservation. It thus fails at large $y$, where a few gluonic 
subcollisions can consume all the available energy or valence quark+gluon subcollisions can consume all the baryon number. For the approach to be valid one has to be far from these limits; there is no problem at RHIC, but at LHC one should not go beyond $|y| \approx 4$.

Another problem is that kinematically a very forward parton demands that the other one also be at large $y$. This, on the other hand, implies that the $x$ value of one of the partons is extremely small and in a region in which the structure functions are unmeasured. This effect is quantitatively discussed in Fig. 4.

\section{Conclusions}

We have in this paper computed the initial number of gluons, quarks and antiquarks with $p_{\mathrm{T}}>p_{0}=2 \mathrm{GeV}$ (value determined by transverse saturation) produced in $\mathrm{Pb}+\mathrm{Pb}$ collisions at $\sqrt{s}=5500 \mathrm{GeV}$ near $y=0$ and studied the further evolution of this system especially in view of the behavior of the net baryon number. The main conclusions are that the produced gluon system is transversally saturated and the parameter $p_{0}$ gets a dynamical significance in LHC nuclear collisions. The perturbatively produced gluon system has also energy/gluon as in a thermal ideal gas, so only isotropisation is needed to make the system thermalised. This clearly enhances possibilities of a very rapid thermalisation. The initial net baryon number density at $\tau_{i}=0.1 \mathrm{fm}$ may be of the order of nuclear matter density, or even larger, but this is rapidly diluted. Initially at $\tau_{i}=0.1 \mathrm{fm}$ the baryon-to-entropy ratio is $(B-\bar{B}) / S \sim 1 / 5000$, and the final baryon-to-entropy ratio may be larger by up to a factor 4 . We conclude that the baryon-to-entropy ratio is small, though still orders of magnitude larger than the corresponding quantity in cosmology.

The main sources of uncertainly in the computation are next-to-leading order corrections, nuclear shadowing of the initial parton distributions and dissipative effects and the non-perturbative source terms during expansion and hadronisation. Nextto-leading order corrections with the acceptance conditions applicable here have not been computed but should be. Nuclear gluon shadowing can only be modelled and ultimately has to be measured. This also holds for dissipative effects. Although the detailed numbers are uncertain, we believe that this general framework will be used to analyse the data when it finally comes.

Acknowledgements We thank D. Kharzeev for reviving our interest in the subject and M. Gyulassy, U. Heinz, L. McLerran, V. Ruuskanen, G. Schuler, and H. Stöcker for useful discussions.

\section{References}



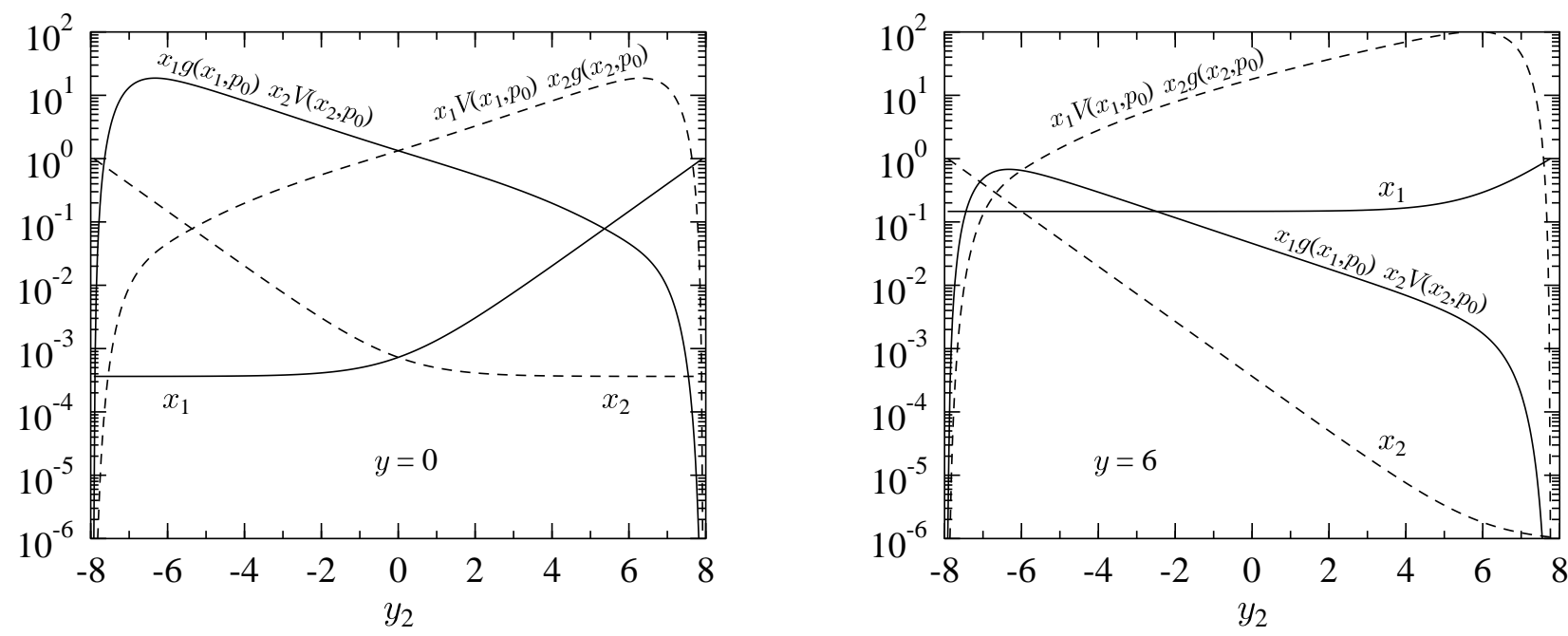

Figure 4: The forward-backward peaking of the quark distribution in Fig. 1 arises from valence-gluon subcollisions. This figure shows the parton luminosities $x_{1} g\left(x_{1}, p_{0}\right) x_{2} V\left(x_{2}, p_{0}\right)$ and $x_{1} V\left(x_{1}, p_{0}\right) x_{2} g\left(x_{2}, p_{0}\right)$ as a function of the rapidity $y_{2}$ of the gluon, which is integrated over in Eq. (1). The $x_{1}$ and $x_{2}$ values of the initial valence quark or gluon are also shown as a function of the rapidity $y_{2}$. For very forward quark rapidities $(y=6)$ the dominant configurations are such in which also the final gluon is at $y_{2} \approx 6$, but then also the $x_{2}$ is extremely small $\left(\sim p_{0}^{2} / s\right)$ and in a region where the structure functions are unknown.

[1] J.-P. Blaizot and A. Mueller, Nucl. Phys. B289 (1987) 847.

[2] K.J. Eskola, K. Kajantie and J. Lindfors, Nucl. Phys. B323 (1989) 37.

[3] K. J. Eskola, K. Kajantie and V. Ruuskanen, Phys. Lett. B332 (1994) 191.

[4] K. J. Eskola and M. Gyulassy, Phys. Rev. C47 (1993) 2329.

[5] D.K. Srivastava, M.G. Mustafa and B. Müller, Preprint DUKE-TH-96-126, nuclth/9608424.

[6] M. Gyulassy, D. Rischke and Bin Zhang, Columbia University preprint CU-TP757, nucl-th/9609030.

[7] K. Geiger and B. Müller, Nucl. Phys. B369 (1992) 600; K. Geiger, Phys. Rev. D47 (1993) 133; Nucl. Phys. A566 (1994) 257c; Phys. Rep. 258 (1995) 237.

[8] T.S. Biró, E. van Doorn, B. Müller, M.H. Thoma and X.-N. Wang, Phys. Rev. C48 (1993) 1275. 
[9] X.-N. Wang and M. Gyulassy, Phys. Rev. D44 (1991) 3501.

[10] X.-N. Wang and M. Gyulassy, Phys. Rev. D45 (1992) 844; Phys. Rev. Lett. 68 (1992) 1480; Comp. Phys. Comm. 83 (1994) 307.

[11] G. Gustafson, in Proc. of Quark Matter '93, eds. E. Stenlund et al., Nucl. Phys. A566 (1994) 233c.

[12] H. Pi, Comp. Phys. Comm. 71 (1992) 173.

[13] K. Werner, Phys. Rep. 232 (1993) 87.

[14] L. V. Gribov, E. M. Levin and M. G. Ryskin, Phys. Rep. 100 (1983) 1.

[15] A. H. Mueller and J. Qiu, Nucl. Phys. B268 (1986) 427.

[16] X.-N. Wang, Phys. Rev. D43 (1991) 104.

[17] T. Sjöstrand and M. van Zijl, Phys. Rev. D36 (1987) 2019.

[18] J. Letessier, A. Tounsi, U. Heinz, J. Sollfrank and J. Rafelski, Phys. Rev. Lett. 70 (1993) 3530 .

[19] J. Cleymans, K. Redlich, H. Satz and E. Suhonen, Z. Phys. C58 (1993) 347.

[20] H. Satz, in Proc. of Quark Matter '91, eds. T.C. Awes et al., Nucl. Phys. A544 (1992) 371c.

[21] U. Heinz, "Heavy ion physics at the LHC", in Proc. of Towards the LHC Experimental Programme, ECFA meeting, 5-8 March 1992, Evian-les-Bains, France, p. 95.

[22] NA49 Collaboration, P. G. Jones et al., Proc. Quark Matter '96, eds. P. BraunMunzinger et al., Nucl. Phys. A, to be published.

[23] D. Kharzeev, Phys. Lett. B378 (1996) 238.

[24] C. Spieles et. al, Phys. Rev. Lett. 76 (1996) 1776.

[25] B.L. Combridge, J. Kripfganz and J. Ranft, Phys. Lett. B70 (1977) 234; E. Eichten, I. Hinchliffe, K. Lane and C. Quigg, Rev. Mod. Phys. 56 (1984) 579; I. Sarcevic, S.D. Ellis and P. Carruthers, Phys. Rev. D40 (1989) 1447.

[26] M. Glück, E. Reya and A. Vogt, Z. Phys. C67 (1995) 433.

[27] K. J. Eskola and X.-N. Wang, in Hard Processes in Hadronic Interactions, eds. H. Satz and X.-N. Wang, Int. J. Mod. Phys. A10, 2881 (1995). 
[28] K.J. Eskola and X.-N. Wang, Phys. Rev. D49 (1994) 1284.

[29] K.J. Eskola, B. Müller and X.-N. Wang, Phys. Lett. B374 (1996) 20.

[30] P. Danielewicz and M. Gyulassy, Phys. Rev. D31 (1985) 53; A. Hosoya and K. Kajantie, Nucl. Phys. B250 (1985) 666.

[31] NA49 Collaboration, S. Margetis et al., Proc. Quark Matter '95, eds. A.M. Poskanzer et al., Nucl. Phys. A590 (1995) 355c. 